\title{
Recognition of Branch Series Resonance Based on Port Equivalent Method
}

\author{
Qunfeng Liu, Yongle Ai, Shuai Li, and Jianying Ding
}

\begin{abstract}
For the identification of series resonance in power system branches, the identification method of branch series resonance based on port equivalent method is proposed. First of all, a typical three-bus test system is used to analyze the series resonance of the branch. It is found that the branch series resonance can be identified by the port equivalent admittance formed between the bus node and the reference node. Then, by establishing the node port association matrix and using the node impedance matrix, the multi-port Thevenin equivalent impedance matrix is obtained, in other words, the equivalent admittance matrix of the port is formed, and the load branch of the node is eliminated, as well as the effect of the node load branch on the series resonance of the identification branch is eliminated. The algorithm effectively utilizes the node admittance matrix commonly used in power system. Finally, the feasibility and correctness of the three-bus system and IEEE9 network system are proved by simulation analysis. The validity of this novel formulation is confirmed through theoretical analysis and case studies.
\end{abstract}

Index Terms-Branch series resonance, equivalent port, node admittance matrix, node impedance matrix.

\section{INTRODUCTION}

$\mathrm{T}$ HE harmonics mainly come from power electronic equipment such as electric arc furnaces and converters. These nonlinear loads produce large amount of harmonics and injects to the grid. The existence of harmonics is inevitable because there is nothing to replace the position of nonlinear loads in the fields of metallurgy and chemical engineering. The impedance of the system is inductive in normal operation of the power system. There are often several series-parallel resonance points, which are affected by the presence of reactive compensation capacitors and high-voltage transmission line capacitors. The operation of the system and the safety of the electrical equipment will be compromised. When the excitation source frequency is equal to the resonance point frequency the resonance will happen in the power system [1]-[3]. It is very meaningful to identify efficiently and comprehensively the existence of series-parallel resonance points in power system.

It's no doubt that the analytical and spectrum analysis methods can accurately identify and determine the series-parallel

Manuscript received April 4, 2019. This work was supported by the National Natural Science Foundation of China under Grant 51777060 and 51277054.

All authors are with the School of Electrical Engineering and Automation, Henan Polytechnic University, Jiaozuo, 454150, China (e-mail: lqf7333@163.com).

Digital Object Identifier 10.24295/CPSSTPEA.2019.00019 resonance point among scholars in the scope of worldwide [4]-[6]. However, the limitations of traditional analytical methods offered have become increasingly emerged for the complex power networks. In [7]-[8], resonance mode analysis (RMA) is proposed, and the impedance, voltage, and current of the system modal can be obtained by decomposing the eigenvalues of the network node admittance matrix, and then decoupling the coupling between the nodes. The identification and analysis of parallel resonance can be done by this method, but it is very difficult for series resonance. Literature [9]-[10] proposed a loop modal analysis method for analyzing series resonance, which is different from the series resonance point identified by spectrum analysis. However, the series resonance point of the loop can be recognized by the method, and it is difficult to identify the series resonance point in the branch. In [11] a series resonance analysis method combining with modal method and virtual branch method is proposed. Based on the idea of node admittance matrix analysis of parallel resonance, the series resonance is analyzed by loop impedance matrix. When a virtual branch is added at each node under the network structure seem as before, the series resonance in the loop is identified by means of eigenvalue decomposition. However, it is difficult to establish a loop impedance matrix in a complex network, and the scale of the matrix impedance will increase a lot with adding a virtual branch. The literature [12]-[13] proposed a branch analysis series resonance, but the resonance point identified by this method is the same as the parallel resonance point identified by the RMA method, which is different from the series resonance point identified by the spectrum analysis method in the paper. Therefore, this method cannot effectively identify the branch series resonance point. In [14], an improved virtual branch method was proposed to analyze series resonance. This method uses the node admittance matrix to analyze the series resonance. It is not necessary to re-establish the loop impedance matrix, but it is still necessary to establish the admittance matrix of the branch and the node relationship matrix. The method is based on a single branch to investigate the series resonance, but whether the parallel resonance or the series resonance is caused by the interaction of components on several branches. Therefore the single branch cannot identify the resonance properties in more detail. A method of using capacitors to scan and identify parallel resonance was proposed [15], which provides a novel solution to the resonance problem of reactive compensation capacitors.

In this paper, the branch series resonance identification method based on port equivalent method is proposed by 


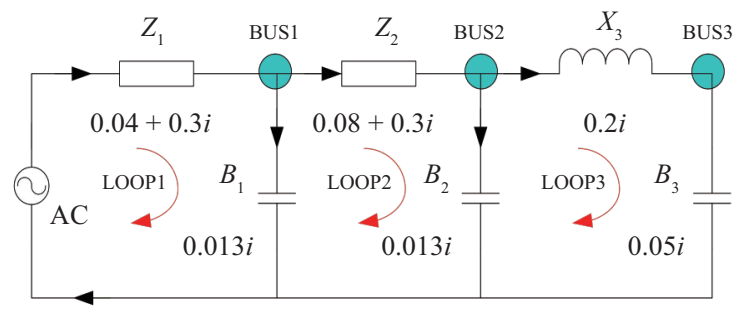

Fig. 1. Three-bus test system.

analyzing the typical branch series resonance identification method. It is found that the branch series resonance can be identified by the port equivalent admittance formed between the bus node and the reference node. This method is implemented through the analysis of the three-bus test system. A multiport Thevenin equivalent impedance matrix is obtained by establishing a node-port association matrix in order that form a port equivalent admittance matrix. The series resonance of the driving point also can be recognized by the over-current, so series resonance frequency can be determined by checking the peak value of the frequency-port equivalent admittance curve. The algorithm effectively utilizes the node admittance matrix commonly used in power systems. Finally, the feasibility and correctness of the proposed method are verified by case study.

\section{Resonance Analysis of Branch SERIES}

The three-bus test system selected is shown in Fig. 1, where the impedance unit is $\Omega$ and the admittance unit is $\mathrm{S}$. Analytical methods are used to solve system series and parallel resonance by the platform of Matlab.

\section{A. Parallel Resonance}

In Fig. 1, the equivalent impedance equations established for the three-bus test system are shown in (1)-(3) respectively (where $f_{\text {base }}=50 \mathrm{~Hz}$ ).

$$
\begin{aligned}
Z_{\text {eqBuS } 1}= & X_{C 1}(h) / / Z_{1}(h) / / \\
& \left\{Z_{2}(h)+X_{C 2}(h) / /\left[X_{3}(h)+X_{C 3}(h)\right]\right\}
\end{aligned}
$$

The harmonic orders calculated for resonant frequency are: $h=4.58,18.96,29.54$.

$$
Z_{\text {eqBus2 }}=X_{C 2}(h) / /\left[Z_{2}(h)+Z_{1}(h) / / X_{C 1}(h)\right] / /\left[X_{3}(h)+X_{C 3}(h)\right]
$$

The harmonic orders calculated for resonant frequency are: $h=4.58,18.96,29.54$.

$$
Z_{\text {eqBus3 }}=X_{C 3}(h) / / X_{3}(h)+X_{C 2}(h) / /\left[Z_{2}(h)+X_{C 1}(h) / / Z_{1}(h)\right]
$$

The harmonic orders calculated for resonant frequency are: $h=4.58,18.96,29.54$. Where: "+" represents the branches connected in series, and "//" represents branches connected in parallel. $Z_{\text {eqBus1 }}, Z_{\text {eqBuS2}}, Z_{\text {eqBuS3 }}$ are the equivalent impedance of BUS1, BUS2 BUS3 respectively; $X_{C 1}(h), X_{C 2}(h)$ and $X_{C 3}(h)$ are the capactiance impedances of the $B_{1}, B_{2}$ and $B_{3}$ of branches respectively; $Z_{1}(h), Z_{2}(h), X_{3}(h)$ are the impedance of the branches respectively; $h$ is the harmonic order.

\section{B. Series Resonance}

There are two types series resonance which are loop series resonance and branch series resonance.

1) Loop series resonance. In Fig. 1, three loop equivalent admittances $Y_{\text {eqLOOP1 }}, Y_{\text {eqLOOP2 }}$ and $Y_{\text {eqLOOP3 }}$ are established as the basic loop based on "mesh" as shown in (4)-(6). The frequencies of series resonance points can be calculated through the equations (4)-(6) respectively.

$$
\left.Y_{\text {eqLOOP1 }}=1 /\left(\begin{array}{l}
Z_{1}(h)+X_{C 1}(h) / / \\
Z_{2}(h)+X_{C 2}(h) / / \\
{\left[X_{3}(h)+X_{C 3}(h)\right]}
\end{array}\right\}\right)
$$

The harmonic orders calculated for resonant frequency are $4.58,18.96,29.54$ respectively.

$$
Y_{\text {eqLoOP2 }}=1 / /\left\{\begin{array}{c}
Z_{2}(h)+X_{C 1}(h) / /\left[Z_{2}(h)\right. \\
\left.+X_{C 2}(h)\right] / /\left[X_{2}(h)+X_{C 3}(h)\right]
\end{array}\right\}
$$

The harmonic orders calculated for resonant frequency are: $h=4.58,18.96,29.54$.

$$
Y_{\text {eqLOOP3 }}=1 / /\left\{\begin{array}{l}
X_{3}(h)+X_{C 3}(h)+X_{C 2}(h) / / \\
{\left[Z_{2}(h)+X_{C 1}(h) / / Z_{1}(h)\right]}
\end{array}\right\}
$$

2) Branch series resonance. In Fig. 1 , assume " $Z_{2}$ " and " $X_{3}$ " as research branch, four branches equivalent admittances $Y_{\mathrm{eq} 1}$, $Y_{\text {eq2 }}, Y_{\text {eq3 } 3}, Y_{\text {eq4 }}$ are established as shown in the equation(7)-(10). The frequencies of series resonance points can be calculated through the equations (7)-(10) respectively.

$$
Y_{\text {eq1 }}=1 /\left[Z_{2}(h)+Z_{1}(h) / / X_{C 1}(h)\right]
$$

The harmonic order calculated for resonant frequency is: $h=22.64$.

$$
Y_{\mathrm{eq} 2}=1 /\left\{Z_{2}(h)+X_{C 2}(h) / /\left[X_{2}(h)+X_{C 3}(h)\right]\right\}
$$

The harmonic orders calculated for resonant frequency are: $h=6.04,26.54$.

$$
Y_{\text {eq3 }}=1 /\left\{\begin{array}{l}
X_{3}(h)+X_{C 2}(h) / / \\
{\left[Z_{2}(h)+X_{C 1}(h) / / Z_{1}(h)\right]}
\end{array}\right\}
$$

The harmonic orders calculated for resonant frequency are: $h=17.68,29$.

$$
Y_{\text {eq4 }}=1 /\left[X_{3}(h)+X_{C 3}(h)\right]
$$

The harmonic order calculated for resonant frequency is: $h=10.00$. 


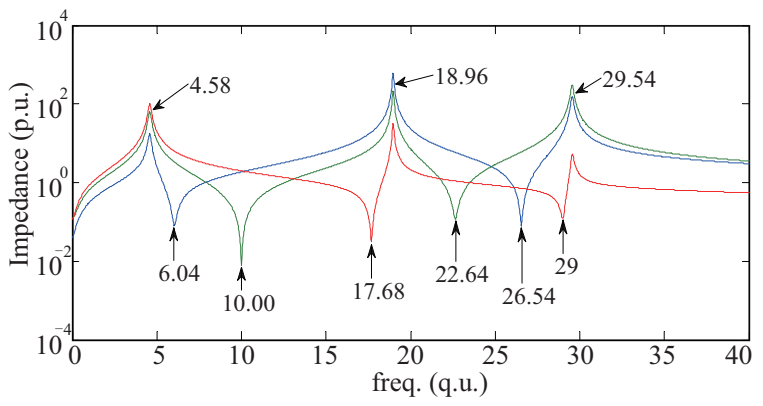

Fig. 2. Frequency impedance curve.

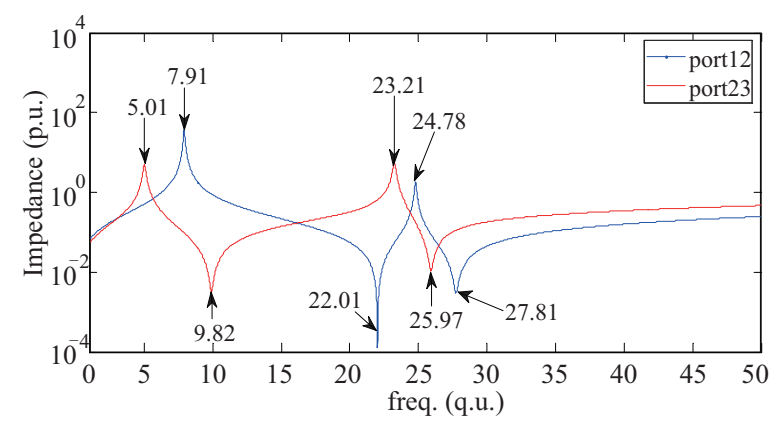

Fig. 3. Frequency impedance curve between non-reference nodes.

It can be known from the above equations. 1) The types of resonance in the system cannot be simply divided into series resonance and parallel resonance. The series resonance needs to be further divided into loop series resonance and branch series resonance; 2) The resonance point from the loop series by the system "mesh" is same as the resonance point from the "node" point which means the parallel sub-circuits.

With Matlab platform the system frequency-impedance diagram is obtained by impedance spectrum scanning simulation as shown in Fig. 2. The parallel resonance points can be determined by checking the peak impedance of the research points and oppositely the series resonance point can be determined by checking the valley impedance of the research points. From Fig. 2, it can be found that in the threebus test system there are three parallel resonance points which are 4.58, 18.96 and 29.54 p.u., and series resonance points which are 6.04, 10.00, 17.68, 22.64, 26.54, 29 p.u.

When the adjacent non-reference nodes are taken as the research objective, the frequency-impedance curve among non-reference nodes is obtained as shown in Fig. 3. Comparing with Fig. 2, it can be seen that when the adjacent non-reference nodes are studied, the parallel resonance point which are 5.01,7.91, 23.21 and 24.78 p.u. and the series resonance points are 9.82, 22.01, 25.97 and 27.81 p.u.. But in the fact we rarely consider the resonance problem between non-reference nodes in the power system, because the non-reference nodes normally are transmission lines. Moreover, when analyzing the resonance problem of the system from the transmission line, it will be different from the inherent resonance points of the system. Therefore, the analysis for the following work of this paper will focus on facts between non-reference nodes and

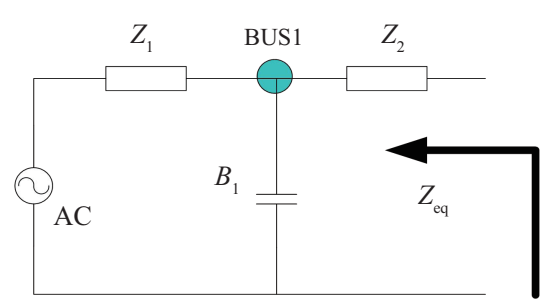

(a)

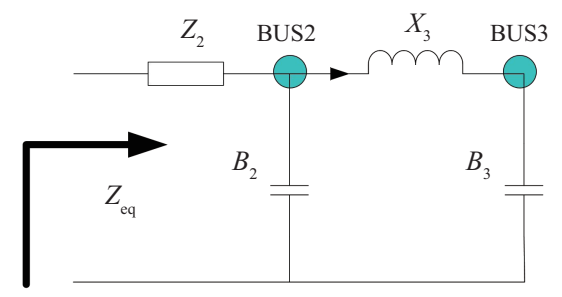

(b)

Fig. 4. Branch series port equivalent diagram.

reference nodes.

\section{Summary}

It is found that the key of the series resonance identification is to select the branch researched. To complete the series resonance identification it will need three steps. First step, a branch researched which is an equivalent port formed between the node and the reference node is selected. Second step, the equivalent Thevenin impedance is calculated. Third step, the equivalent port admittance is obtained by converting the equivalent Thevenin impedance calculated. Taking the branch " $Z_{2}$ " shown in Fig. 1 as an example, the corresponding branch series resonant port is shown in Fig. 4.

As can be seen from Fig. 4, for a system with $N$ independent nodes, the number of ports for the series resonance of the branch is $2 N-2$. When the system is a ring network, the amount of the ports is $2(N-1) \sim 2 C_{N}^{2}$ because the amount of branches is $(N-1) \sim C_{N}^{2}$ in a ring network with $N$ independent nodes. If the non-reference node is used as the research objective, the amount of ports for the series resonance of the branch is $N^{*}(N-1) / 2$. When only adjacent non-reference nodes are considered, the corresponding amount of ports for identifying the series resonance of the branch is $N-1$.

\section{Port Equivalent Method}

A power network with $N$ nodes (excluding reference nodes) [16] is shown in Fig. 5. The $m$ ports (port subscripts denoted by $a, b, \ldots, m)$ are taken into account, the node-port correlation matrix can be established. Then a multi-port Thevenin equivalent port impedance matrix of $m \times m$-dimension formed is analyzed. The Norton equivalent port admittance matrix can be obtained by inversing the Thevenin equivalent port impedance matrix.

In Fig. 5, $p, q$ are two nodes which are formed $a$ port. The direction of the current is from first node to the second node 


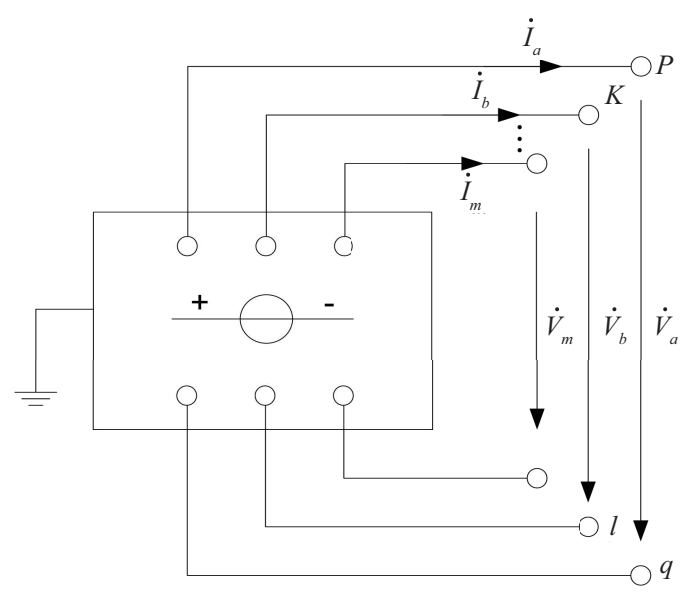

Fig. 5. Power network.

which means $p \rightarrow q$. If neither $p$ nor $q$ in $a$ port is reference node, the $N \times 1$ dimensional correlation vector of the nodeport is,

$$
M_{a}=\left[\begin{array}{lllllll}
0 & \cdots & 1 & \cdots & -1 & \cdots & 0
\end{array}\right]^{T}
$$

where " 1 " and " -1 " represents the positions of the $p$ th and $q$ th.

If the node of $q$ is a reference node, its corresponding $N \times 1$ dimensional node-port correlation vector is,

$$
M_{a}=\left[\begin{array}{lllllll}
0 & \cdots & 1 & \cdots & 0 & \cdots & 0
\end{array}\right]^{T}
$$

The node-port correlation vectors formed by the $m$ ports are put together in columns to form a $N \times m$ dimensional node-port correlation matrix $M$.

$$
M=\left[\begin{array}{llll}
M_{a} & M_{b} & \cdots & M_{m}
\end{array}\right]
$$

Therefore, the formed $m \times m$-dimensional multiport Thevenin equivalent port impedance matrix is:

$$
Z_{\text {eq }}=M^{T} \cdot Z \cdot M
$$

where $Z$ is the network impedance matrix.

Assume that the $m$ ports of the original network in Fig. 4 are opened, the equivalent electromotive voltage of the Thevenin equivalent port are,

$$
\dot{V}_{\text {eq }}^{(0)}=M^{T} \dot{V}^{(0)}=\left[\begin{array}{llll}
\dot{V}_{a}^{(0)} & \dot{V}_{b}^{(0)} & \cdots & \dot{V}_{m}^{(0)}
\end{array}\right]
$$

where $M^{T}$ is the transposed matrix of the node-port association matrix; $\dot{V}^{(0)}$ is the open circuit voltage vector of the port; $\dot{V}_{a}^{(0)}$ $\dot{V}_{b}^{(0)} \ldots \dot{V}_{m}^{(0)}$ are the port open circuit voltage of $a, b, \ldots, m$, respectively.

Then, Thevenin equivalent network of $m$ ports can be

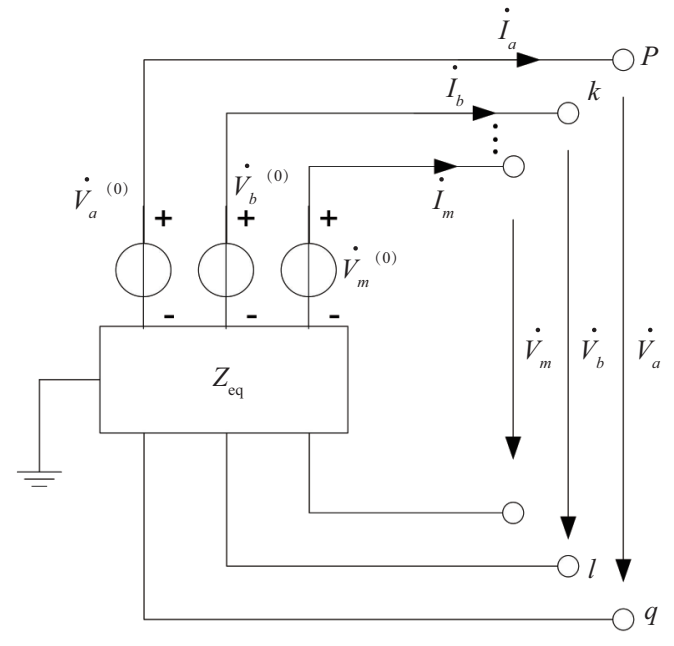

Fig. 6. $m$ port's Thevenin equivalent network.

formed, as shown in Fig. 6 .

It is known from (14) that the multi-port Norton equivalent port admittance matrix of the $m \times m$-dimensional is,

$$
Y_{\mathrm{eq}}=Z_{\mathrm{eq}}-1
$$

For the three-bus system shown in Fig. 1, a port correlation matrix formed by its corresponding node and a reference node can be established.

$$
M=\left[\begin{array}{lll}
1 & 0 & 0 \\
0 & 1 & 0 \\
0 & 0 & 1
\end{array}\right]
$$

It is known from (14) and (17) that the original network is a multi-port Thevenin equivalent circuit.

Also it is known from (7)-(10) that when the port equivalent admittance is formed, the branch of the corresponding node is not considered. Therefore, in order to eliminate the influence of the node branch on the identification branch series resonance, numerical processing means is used. The flow chart of the port equivalent method for eliminating the branch is shown in Fig. 7.

Taking the single-bus test system as the research objective, as shown in the Fig. 8. When the branch 1\# is not eliminated, the parallel resonance resonant frequency is 13.38 p.u. and the series resonance resonant frequency is 24.32 p.u.. When the branch $1 \#$ is eliminated, the resonance in the system is the series resonance of the branch, and the frequency of the series resonance point of the branch is 24.32 p.u.. Compared the results of the eliminating the branch 1 \# before and after there are no any difference for the identification of series resonance points in the study case.

For the three-bus test system shown in Fig. 1, the TheveninNorton port equivalent method proposed in this paper is used to analyze the branch series resonance. The result is shown in Fig. 9. The six-branch series resonances are 6.04, 10.00, 17.68, 


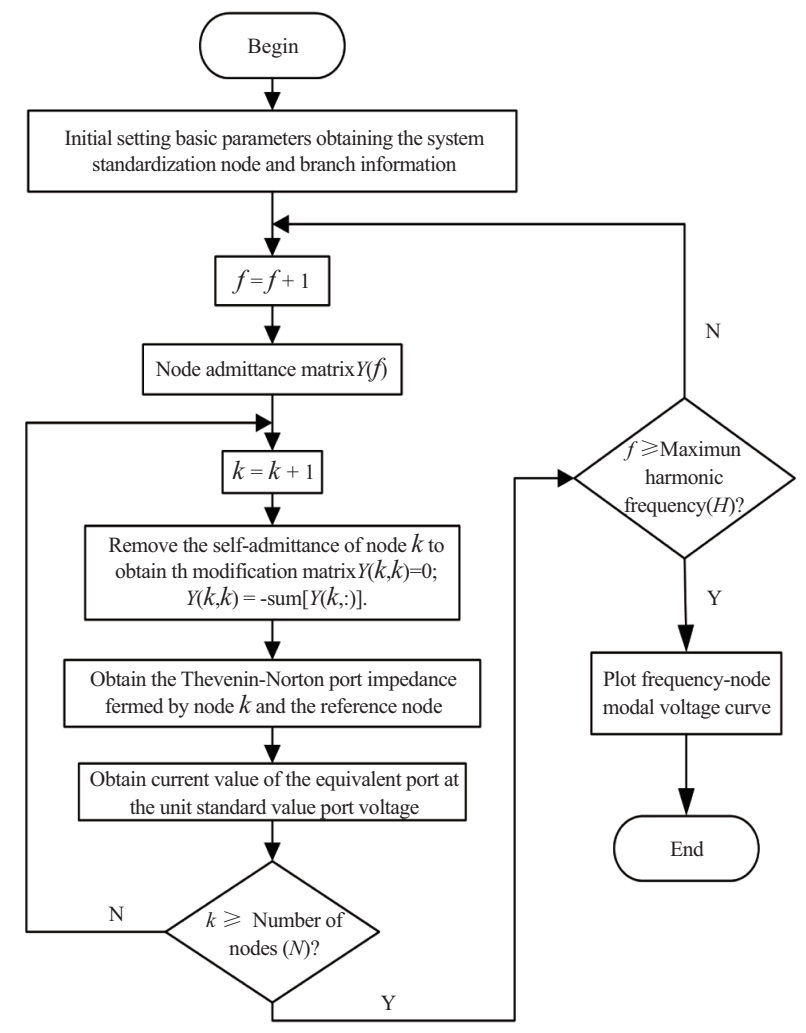

Fig. 7. Algorithm flow chart.

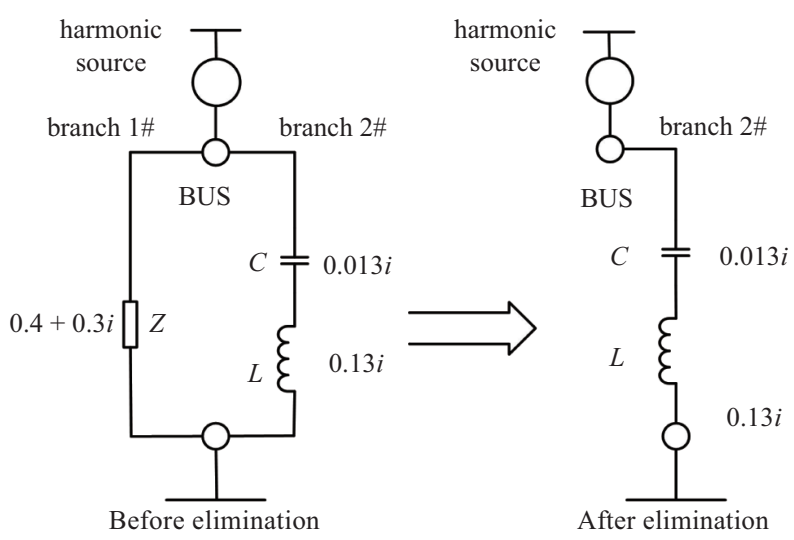

Fig. 8. Single-bus test system.

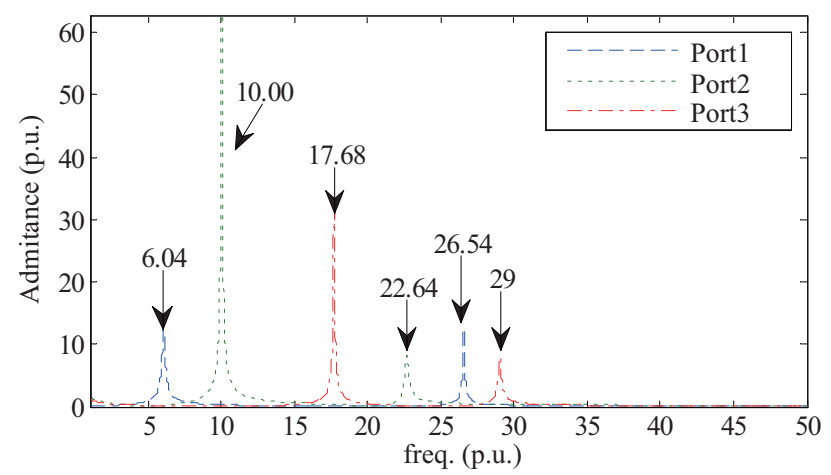

Fig. 9. Equivalent port method in series resonance recognition of branch line.

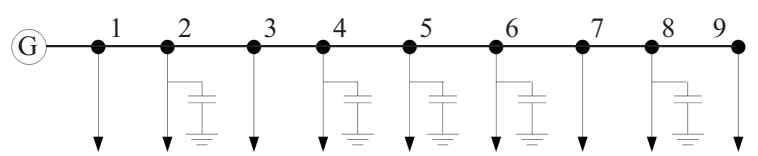

Fig. 10. IEEE9 system diagram.

TABLE I

IEEe9 Distribution Network System Parameter

\begin{tabular}{cccccccc}
\hline \hline $\begin{array}{c}\text { Branch } \\
\text { number }\end{array}$ & $\begin{array}{c}\text { First } \\
\text { node }\end{array}$ & $\begin{array}{c}\text { End } \\
\text { node }\end{array}$ & $R$ (p.u.) $X$ (p.u.) & $\begin{array}{c}\text { End } \\
\text { work } \\
(\mathrm{kW})\end{array}$ & $\begin{array}{c}\text { End reactive } \\
(\mathrm{kVar})\end{array}$ & $\begin{array}{c}\text { Capacitor } \\
\text { number }\end{array}$ \\
\hline 1 & 0 & 1 & 0.0233 & 0.0780 & 1840 & 460 & 0 \\
2 & 1 & 2 & 0.0026 & 0.1144 & 980 & 340 & 5 \\
3 & 2 & 3 & 0.1411 & 0.2278 & 1790 & 446 & 0 \\
4 & 3 & 4 & 0.1320 & 0.1150 & 1598 & 1840 & 2 \\
5 & 4 & 5 & 0.3749 & 0.3266 & 1610 & 600 & 4 \\
6 & 5 & 6 & 0.1711 & 0.1491 & 780 & 440 & 5 \\
7 & 6 & 7 & 0.3885 & 0.2200 & 1150 & 60 & 0 \\
8 & 7 & 8 & 0.9065 & 0.5134 & 980 & 130 & 5 \\
9 & 8 & 9 & 1.0101 & 0.5721 & 1640 & 200 & 0 \\
\hline \hline
\end{tabular}

22.64, 26.54, 29 p.u. respectively. Meanwhile, "Port 1" in Fig. 9 represents the self-impedance of the node 1st that is removed, and the branch series resonance identification between the node 1 and the reference node (the ground node) can be carried out.

It can be also known from Fig. 9 that the proposed algorithm can effectively identify the branch series resonance.

\section{CASE Studies}

In order to further verify the feasibility and effectiveness of the proposed Thevenin-Norton equivalent port method to identify the branch series resonance, the IEEE9 node distribution network system shown in Fig.10 is selected for simulation analysis.

For the load of each node in the IEEE9 network system shown in Fig. 10, the high, middle and low load states are given in [15]. The middle state load is selected as the research objective. The case of a set of optimized capacitor configurations is selected and used into the network. The location of the capacitors is indicated in Fig. 10. The parameters of each node load, line impedance, and capacitor configuration of the IEEE9 network system are shown in Table I.

For the IEEE9 node network system, the standard capacity is $100 \mathrm{MVA}$, and a single capacity of the configured capacitor is $300 \mathrm{kVar}$. At the same time, the load parameters of each node are modeled as series constant impedance load equivalent. As comparing the node 4th as shown in Fig. 10 is selected. The frequency series analysis method and the method proposed in this paper are used to identify the branch series resonance, and the branch series resonance point identification map is shown in Fig. 11. 


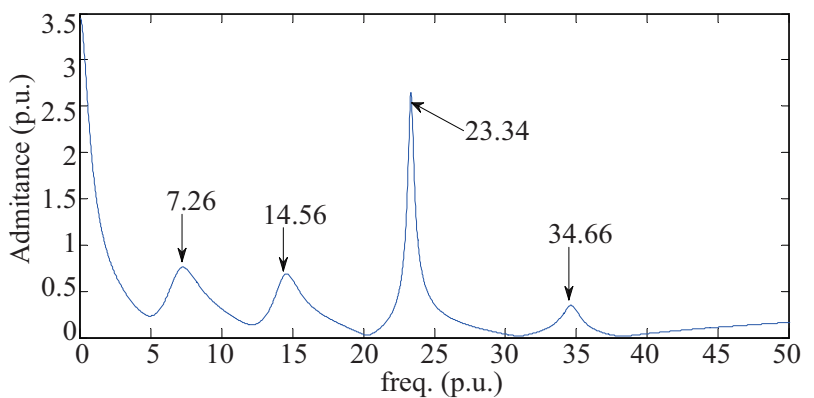

(a)

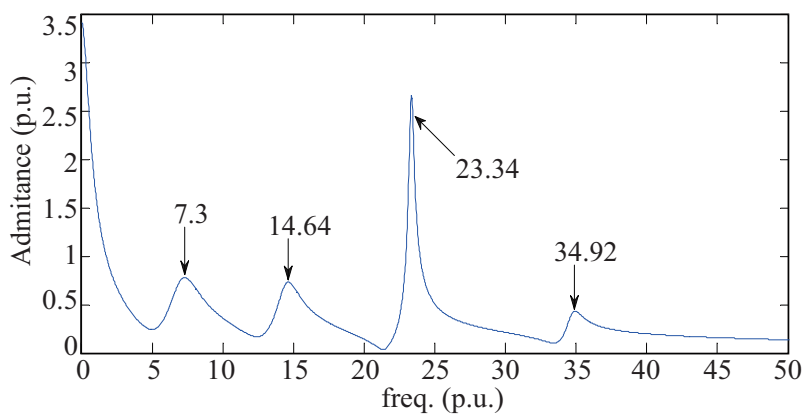

(b)

Fig. 11. IEEE9 system branch series resonance recognition diagram. (a) Spectrum analysis. (b) Equivalent port method.

It is known from Fig. 11 that four branch series resonances are identified by spectrum analysis. All of four branch series resonances are identified by using the equivalent port method, which proves the feasibility of the algorithm.

\section{CONCLUSION}

The series-parallel resonance is divided into two types of the resonance problem, but the resonance in the power system cannot be simply divided into parallel resonance and series resonance. For series resonance, it can be divided into loop series resonance and branch series resonance. For parallel resonance, the modal method based on the node admittance matrix can be used to identify and analysis. For the series resonance of the branch, it cannot be simply regarded as a loop series resonance to identify the series frequency. The resonance can be identified using the port equivalent admittance formed by the node corresponding to the branch. Therefore, an equivalent port method is proposed to identify the branch series resonance using the three-bus test system, it is found that the branch series resonance can be identified by the port equivalent admittance formed between the bus node and the reference node. By establishing the node-port correlation matrix and using the node impedance matrix, the multi-port Thevenin equivalent correlation matrix is obtained. And then the port equivalent admittance matrix can be obtained. By using characteristic of the frequency-port equivalent admittance the branch series resonance frequency point can be determined and is equal to the peak point frequency of the frequency-port equivalent admittance curve.

\section{REFRENCES}

[1] B. Julio, D. RamonI, and A. Matilde. "Applications of wavelet transform for analysis of harmonic distortion in power systems: A review," IEEE Transactions on Transaction and Measurement, vol. 61, no.10, pp. 857-861, Oct. 2012.

[2] M. Esmaili, H. Shayanfar, and A. Jalilian. "Modal analysis of power systems to mitigate harmonic resonance considering load models," Energy, vol. 33, no. 9, pp. 1361-1368, Sep. 2008.

[3] H. Sharma, R. Matthew, and D. Doug. "Grid impacts due to increased penetration of newer harmonic sources, "IEEE Transactions on Industry Applications, vol. 52, no. 1, pp. 99-104, Jan. 2016.

[4] A. Dolara, M. Gualdoni, and S. Leva. "Impact of high-voltage primary supply lines in the $2 \times 25 \mathrm{kV}-50 \mathrm{~Hz}$ railway system on the equivalent impedance at pantograph terminals," IEEE Transactions on Power Delivery, vol. 27, no. 1, pp.164-175, Jan.2011.

[5] J. Wu, W. Han, T. Chen, J. Q. Zhao, B. B. Li, and D. G. Xul. "Resonance characteristics analysis of grid-connected inverter systems based on sensitivity theory," Journal of Power Electronics, vol. 18, no. 3, pp. 746-756, May 2018.

[6] Z. K. Shuai, D. G. Liu, J. Shen, C. M. Yu, Y. Cheng, and A. Luo. "Series and parallel resonance problem of wideband frequency harmonic and its elimination strategy," IEEE Transactions on Power Electronics, vol. 29, no. 4, pp. 1941-1952, Apr. 2014.

[7] Y. Cui and W. S. Xu, "Harmonic resonance mode analysis using real symmetrical nodal matrices," IEEE Transactions on Power Delivery, vol. 22, no. 3, pp. 1989-1990, Jul. 2007.

[8] C. Amornvipas and L. Hofmann. "Resonance analyses in transmission systems: experience in germany," IEEE PES General Meeting, pp.18, 2010.

[9] C.X.Yang, K.P.Liu, and D.X.Wang "A loop modal analysis method for series harmonic resonance assessment," High Voltage Engineering, vol. 34, no.11, pp.2459-2462, Nov. 2008.

[10] H. T. Hu, Z. Y. He, Y. F. Zhang, and S. B. Gao. "Modal frequency sensitivity analysis and application using complex nodal matrix,"IEEE Transactions on Power Delivery, vol. 29, no. 2, pp. 69-971, Apr. 2014.

[11] H. Zhou, Y. W. Wu, S. H. Lou, and X. Y. Xiong. "Series resonance analysis based on modal analysis and dummy branch method," Proceedings of the Chinese Society of Electrical Engineering, vol. 27, no. 28, pp. 84-89, Oct. 2007.

[12] H. T. Hu, Z. Y. He, M. Zhang, S. B. Gao, and Q. Q. Qian. "Series resonance analysis in high-speed railway all-paralle AT traction power supply system,"Proceedings of the Chinese Society of Electrical Engineering, vol. 32, no. 13, pp. 52-60, May 2012.

[13] B. Wang, X. F. Jiang, S. B. Gao, and Z. C. Qiu, "Series resonance analysis based on branch-circuit method," Journal of Southwest Jiaotong University, vol. 47, no. 6, pp.1021-1026, Dec. 2012.

[14] B. Wang, Z. C. Qiu, X. D. Han, X. F. Jiang, and S. B. Gao, "Series resonance analysis based on improved dummy branch method for high-speed railway traction network," Electric Power Automation Equipment, vol. 35, no. 11, pp. 90-95, Nov. 2015.

[15] G. T. Heydt, H. U. Patil, J. Loehr, and T. Larose. "Harmonic resonance assessment for transmission class shunt capacitors" Proceedings of the IEEE Power Engineering Society Transmission and Distribution Conference, pp. 1-5, Jul. 2016.

[16] A. A. R. Quete, J. E. C. Navarro, H. C. Zini, and G. Ratta, "Frequency scan analysis supported by a possibility theory-based approach," International Transactions on Electrical Energy Systems, vol. 28, no. 11, pp.90-95, Nov. 2018. 


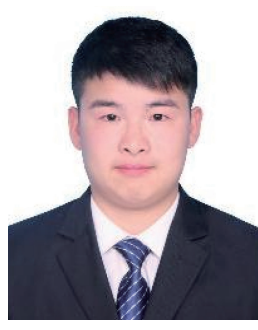

Qunfeng Liu was born in China. He received the B.S. degree in Electrical Engineering and Automation from Henan Polytechnic University, Jiaozuo, China, in 2017. He is currently pursuing M.S. degree in Electrical Engineering and Automation at Henan Polytechnic University. His current research interests include power system analysis and micro-grid control. M.S. Liu is a member of Chinese Association for Artificial Intelligence.

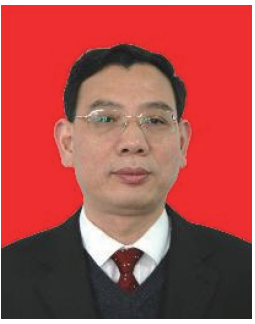

Yongle Ai was born in China. He received the B.S. degree in Power Electronics from Xi'an University of Technology, Xi'an, China, in 1987, received the M.S. degree in Electrical Engineering from Southwest Jiaotong University, Chengdu, China, in 1995, and the Ph.D. degree from the University of Stellenbosch, Stellen-bosch, South Africa, in 2006.

He joined the Henan Polytechnic University from 1987 to now. He is currently a Professor at School of Electrical Engineering and Automation, Henan Polytechnic University, China. His research interests include multiphase $\mathrm{AC}$ machine control and power system filter. Professor Ai is a member of Chinese Society for Electrical Engineering.

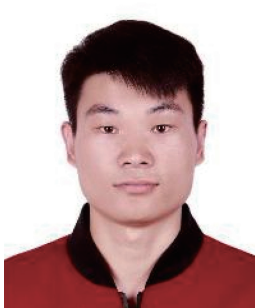

Shuai Li was born in China. He received the B.S. degree in Electronics and Electrical from Henan Normal University, Xinxiang, China, in 2015, received the M.S. degree in Electrical Engineering and Automation from Henan Polytechnic University, Jiaozuo, China, in 2019. Now he is an engineer at Zhengtai Electric. His current research interests include power system analysis and system optimization analysis.

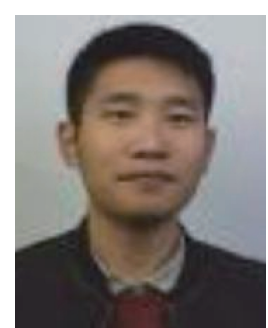

Jianying Ding was born in China. He received the B.S. degree in Electrical Engineering and Automation from Henan Polytechnic University, Jiaozuo, China, in 2017. He is currently pursuing M.S. degree in Electrical Engineering and Automation at Henan Polytechnic University. His current research interests include micro-grid control and optimization scheduling. 